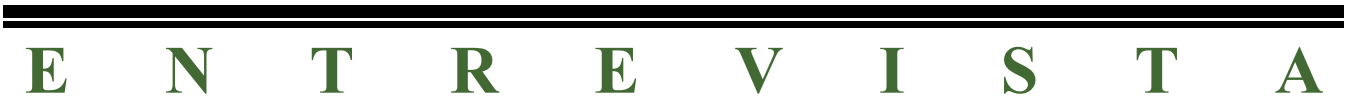




\section{ENTREVISTA A RENATO TREVES}

1.

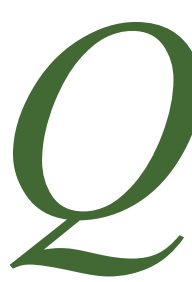

uisiéramos empezar con algunos apuntes biográficos. Sus primeros anos de vida académica coinciden con un momento particularmente interesante de la universidad italiana. Tenemos entendido que entre sus maestros se contaron Gioele Solari y Rodolfo Mondolfo y que fue uested condiscípulo de Bobbio. ¿Qué importancia tuvieron para usted las enseñanzas de Solari y Mondolfo? ¿Qué otros profesores de esa etapa tuvieron para usted un interés especial? ¿Cómo incidía el clima espiritual impuesto por el fascismo en la vida académica? ¿Bajo qué circunstancias decidió usted emigrar a la Argentina? ¿Qué acogida se le dispensó allí?

Por lo que se refiere a mis datos biográficos y a mis primeros años de vida académica, diré que nací en Turín el 6 de noviembre de 1907 y que, después de haber frecuentado el ginnasio-liceo Massimo d'Azeglio, me matriculé en el primer curso de la Facultad de Derecho de la Universidad de Turín para el año académico 1925-26, el año en que, después del asesinato de Matteotti, el gobierno fascista promulgó las leyes especiales que instauraron y consolidaron la dictadura. Mi orientación cultural y política estaba ya, pues, bastante clara en aquel año, pero se hizo más segura y precisa en el año siguiente, 1927-28, en el que tuve tres profesores de extraordinario relieve: Francesco Ruffini, profesor de Derecho eclesiástico y senador que se había opuesto valientemente en el Senado a la promulgación de algunas leyes fascistas; Luigi Einaudi, profesor de ciencia de las finanzas, también él senador liberal antifascista y que, como es sabido, tras el final de la dictadura sería presidente de la República; y, en fin, Gioele Solari, profesor de filosofía del Derecho que, aun estando lejos de la política militante, había ejercido y ejercía una particular fascinación sobre los jóvenes antifascistas. Y no puedo olvidar que en ese año establecí lazos de profunda amistad, una 
amistad, una amistad que duró toda la vida, con dos compañeros que jugaron después un papel del máximo relieve en la lucha contra el fascismo y en la Resistencia: Mario Andreis y Aldo Grosci.

Mi maestro fue Gioele Solari. Con él leí en 1929 mi tesis de licenciatura sobre «La doctrina saintsimoniana en el pensamiento italiano del Resurgimiento». Un tema que respondía a mis intereses juveniles por la tradición republicana del Resurgimiento y por el socialismo naciente. Después de haber publicado aquella tesis, en 1931, cuando decidí dedicarme a los estudios de filosofía del Derecho, fue Solari quien me aconsejo que me ocupara del neokantismo y que efectuase un viaje de estudios a Alemania; desde entonces, y por muchos años, en las diversas vicisitudes de mi vida él fue siempre para mí el punto de referencia seguro, el consejero paterno y afectuoso.

No conocí personalmente a Mondolfo en el período anterior al exilio argentino. En ese período tuve con él sólo una relación epistolar a propósito de mi ensayo sobre el saintsimonismo, ensayo que le había enviado por sugerencia de Alessandro Levi, profesor de filosofía del Derecho en la Universidad de Parma y muy vinculado a él. Con Levi, por el contrario, tuve relaciones de devota amistad desde los años universitarios. Me había puesto en relación con él mi sobrino Paolo Treves, hijo de Claudio Treves, diputado socialista por Milán. Después de la expatriación de su padre, Paolo, con su madre (la señora Oiga, hermana de Levi), había ido a establecerse a Turín y en aquella universidad estaba también él preparando su tesis sobre el pensamiento político de Campanella, bajo la dirección del maestro común, Solari. Desde el primer momento me sentí conmovido por la cordialidad, la vivacidad y la sencillez de Levi, el ilustre estudioso que desde entonces, y después, para toda la vida, quiso demostrarme su amistad. Recuerdo sus palabras de ánimo con ocasión de mi venia docente y mi primer encargo en la Universidad de Urbino. Recuerdo las ayudas y los consejos que me dio antes de mi partida para la Argentina. Y recuerdo, en fin, todo cuanto hizo después de la Liberación para que yo pudiera volver a enseñar en Italia y ser su sucesor en la Universidad de Parma.

No fui condiscípulo de Bobbio. Mi primer encuentro con él tuvo lugar en 1931, en el Instituto Jurídico de la Universidad de Turín, cuando yo era ya licenciado desde hacía un par de años y él estaba por licenciarse con una tesis sobre el idealismo italiano dirigida por nuestro común maestro Solari. Los lazos de amistad entre nosotros se establecieron inmediatamente y se consolidaron en el año siguiente, 1932, cuando ambos, habiendo decidido 
dedicarnos al estudio de la filosofía alemana, por consejo de Solari, nos fuimos en viaje de estudios a la Alemania aún democrática. De vuelta a Turín iniciamos el camino habitualmente seguido para la carrera universitaria. En primer lugar, el examen para la venia docente que superamos en 1934. Posteriormente, el encargo en las dos universidades más pequeñas de Italia en aquel tiempo y, concretamente: Bobbio en Camerino y yo en Urbino. En fin, en 1938, el esperado concurso a la cátedra. Concurso que ganó Bobbio, quien poco después fue llamado por la Universidad de Siena, y del que yo fui excluido al resultar afectado por las leyes racistas promulgadas en septiembre de aquel año y que me privaban de toda posibilidad de trabajo y de vida digna en mi país.

Personalmente, tengo que decir que dichas leyes no fueron acogidas por mí de manera dramática. Desde hacía tiempo tenía la sensación precisa de lo que iba a suceder, y la decisión de emigrar a un país que me ofreciera la posibilidad de vivir y de trabajar fue natural e inmediata. También tuvo su parte el espíritu de aventura, un cierto deseo de revancha y quizás también el hecho de que la idea de emigrar no era para mí, al menos en teoría, una idea totalmente nueva.

La elección de trasladarme a América latina se debió a varios motivos. Razones de carácter contingente: una presentación de Eugenio Florian, que había sido mi profesor de Derecho penal en Turín, al profesor Salvagno Campos, de Montevideo, quien tenía una gran devoción por el primero, y el nombramiento como miembro del «Instituto argentino de filosofía jurídica y social» que me llegó precisamente en esos días; nombramiento que me hizo pensar que en ese país habría de encontrar a alguien que me conociera. Además, motivos de carácter general: por un lado, la afinidad de la lengua y el carácter de los habitantes que habría de hacer más fácil la adaptación; por otro lado, la fascinación de la cultura española republicana que pensaba estaría viva y activa en aquellos lejanos países que se mostraban dispuestos a acoger a los prófugos de la guerra civil.

Embarqué en Nápoles hacia el final de octubre de 1938 y, después de dieciocho días de navegación (entonces no se hablaba de viajes aéreos), desembarqué en Montevideo, pero no encontré posibilidades inmediatas de establecerme. Proseguí, pues, a Buenos Aires donde, después de haber participado en una reunión del Instituto argentino al que me he referido, con el apoyo del presidente del mismo, Carlos Cossio, que era profesor en la Plata pero tenía mucha influencia en Tucumán, su ciudad natal, obtuve un «contrato anual» para enseñar Introducción al Dere- 
cho en aquella universidad, enseñanza que inicié en mayo de 1939 y concluí al final de aquel año académico, es decir, cuando obtuve, para el año sucesivo, una cátedra de sociología en la Facultad de Filosofía y Letras de la misma universidad.

2. Durante su estancia en Argentina tuvo usted oportunidad de entrar en contacto con el pensamiento español del exilio. Se ha referido usted con frecuencia a la influencia que supuso para usted la filosofía de Ortega y Gasset y ha aludido también en alguna ocasión al aprecio que siente por la obra de algunos juristas españoles, como Jiménez de Asúa. ¿Podría detallarnos en qué medida y en qué aspectos la filosofía de Ortega y, en general, el contacto con el pensamiento español y latinoamericano han sido importantes para usted?

Para contestar a esta pregunta, ante todo me parece oportuno recordar que «la fascinación por la cultura española republicana» de que he hablado no se debía únicamente a la simpatía que despertaba en mí el país que en 1936 había resistido a la agresión franquista y que después se había comprometido en una guerra desesperada contra el fascismo propagado en Europa, sino que se debía también a recuerdos e impresiones personales. En septiembre de 1937 me había encontrado clandestinamente en París con Garosci que regresaba de aquella guerra, y en nuestras conversaciones me había hablado no sólo de las acciones militares en las que había participado, del asesinato de los hermanos Roselli o de los problemas de la política italiana e internacional, sino que me había hablado también de la cultura española de la que era un sincero admirador, de poetas como Federico García Lorca y Rafael Alberti, de filósofos como José Ortega y Gasset, de filólogos como Américo Castro y de historiadores como Claudio Sánchez Albornoz.

Es fácil imaginar la impresión que experimenté cuando llegué a Buenos Aires en diciembre de 1938 y me encontré, llegados con dificultad como emigrados a esa ciudad, a algunos exponentes de la cultura española de la que Garosci me había hablado con tanto entusiasmo. Y uno de los hechos que me impresionó desde el principio fue el lazo que se había establecido inmediatamente entre estos exiliados y los intelectuales argentinos más abiertos y responsables y del que pude darme cuenta desde los primeros días de mi llegada frecuentando la editorial Losada en la que me había introducido Cossio, quien dirigía en la misma una colección de filosofía del Derecho en la que me había invi- 
tado a colaborar. Conocí así en esa editorial a algunos intelectuales españoles de la generación anterior a la mía, como Luis y Felipe Jiménez de Asúa y a otros de mi propia generación, como el sociólogo Francisco Ayala y el economista Jesús Prados Arrarte, y conocí también a algunos intelectuales argentinos de notable nivel, entre los que estaba Francisco Romero, el filósofo más apreciado del momento, sostenedor de una dirección de pensamiento propia, pero muy abierto hacia la filosofía de Ortega y Gasset y hacia la filosofía alemana de la cultura. Y del significado y de la importancia del vínculo entre cultura española y argentina, que había notado desde los primeros días de mi llegada a Buenos Aires, pude hacerme después una idea cada vez más precisa durante los largos años transcurridos en Tucumán, donde tuve colegas españoles como el pedagogo Lorenzo Lusuriaga y el filólogo clásico Clemente Balmori, junto con los colegas argentinos discípulos de Romero: Eugenio Pueciarelli, Aníbal Sánchez Reulet y los hermanos Silvio y Risieri Frondili.

Para explicar la importancia que tuvo par mí el contacto con el pensamiento español y argentino, tengo que recordar ahora hasta donde habían llegado mis estudios de fílosofía del Derecho cuando salí de Italia. Entre 1933 y 1934, después de mi vuelta de Alemania, había publicado algunos trabajos sobre la filosofía jurídica neokantiana en los que concluía compartiendo las críticas que habían dirigido al formalismo kelseniano autores que, como Heller o von Hippel, sostenían que la ciencia del Derecho tenía que adherirse cada vez más a las exigencias de la vida y de la historia y tenía que rellenar de contenido concreto las simples formas conceptuales. Posteriormente, desde 1934 a 1938, siguiendo la vía de la crítica al formalismo, me había ocupado del problema de la experiencia jurídica, esperando encontrar en las doctrinas que habían profundizado en ese problema, una dirección de pensamiento en la que poder encontrar una referencia; pero tengo que decir que en doctrinas tales como las de Schuppe, Capograssi, Gurvitch y otros autores que había tomado en consideración, no había encontrado soluciones satisfactorias.

En cambio, consideré que podía encontrar tales soluciones en la filosofía de la cultura de que hablaban Romero, Ortega y Gasset y, obviamente, los autores alemanes a que éstos hacían referencia. Así, en la Introducción a un ensayo titulado «Diritto e cultura» en que había examinado ampliamente las doctrinas de estos autores, pude sintetizar los resultados alcanzados diciendo que los mismos consistían «esencialmente en afirmar la exigencia de entender la experiencia jurídica como una experiencia cultural y en plantear y profundizar el problema del Derecho fraccio- 
nándolo y proyectándolo en el más vasto campo de los fenómenos culturales.

En Ortega y en algunos otros autores considerados por mí en ese ensayo y también en ensayos posteriores, valoraba su relativismo y su perspectivismo que no llevaban hacia las posiciones del escepticismo, sino, por el contrario, hacia las del liberalismo. Así, puedo recordar que, para Ortega, con el perspectivismo se derrumba la errónea pretensión de que la realidad tendría «una fisonomía propia, independientemente del punto de vista desde la que se considera» y, por tanto, también la creencia de que «toda visión de la realidad desde un punto de vista determinado sería falsa si no coincide con su aspecto absoluto». Y a esta creencia le sustituye la convicción opuesta, propia del liberalismo, según la cual, la realidad se compone por su íntima naturaleza «de infinitas perspectivas todas igualmente verdaderas y auténticas; la única perspectiva falsa es la que pretende ser la única verdadera».

Como ya he dicho, no conocía a Mondolfo antes de mi partida de Italia. Lo conocí a su llegada a Buenos Aires en 1939, llegada que me había sido preanunciada por nuestro común amigo Levi. Mondolfo no ha sido, pues, formalmente mi maestro, pero lo fue de hecho en cuanto que durante mi estancia en la Argentina y también después estuvo siempre afectuosamente próximo y aprendí mucho de él y de su obra. Más concretamente, en sus escritos encontré una distinta pero no menos interesante filosofía de la cultura ligada a posiciones relativistas y problematicistas. Entre otros, pienso en su libro «Alle origini della filosofia della cultura» y en el ensayo final que se contiene, donde, después de haber afirmado que «la filosofía representa la cultura humana que adquiere consciencia de sí», pone de manifiesto el problematicismo de la filosofía que siempre sostuvo, al afirmar que «la consciencia filosófica... no es tanto el conocimiento de los sistemas cuanto la consciencia de los problemas» y que para la filosofía «la formación de los sistemas es, en todo momento, una exigencia de la consciencia de los problemas».

Por lo que se refiere a los años de mi permanencia en Argentina, tengo que decir que en ellos no me ocupé únicamente del Derecho como fenómeno cultural, sino también de otros problemas. Como profesor de sociología, después de haber publicado en 1941 un libro teórico titulado «Sociología y filosofía social», realicé algunas investigaciones empíricas sobre los conventillos (las viviendas de los cortadores de caña) en Tucumán y publiqué también en 1942 un librito titulado «Introducción a las investigaciones sociales». Además, me ocupé de las relaciones 
entre el pensamiento italiano y el argentino, publicando un trabajo sobre la influencia de Vico en la filosofía jurídica de saintsimonianos Alberti y otro trabajo sobre los italianos y argentinos en los países del Río de la Plata. Por lo demás, preparé la edición argentina de los dos volúmenes de Gioele Solari titulados «La idea individual y la idea social en el Derecho privado». En fin, publiqué un libro sobre el pensamiento político de Benedetto Croce titulado «Croce, filósofo de la libertad», en el que traté de establecer una vinculación entre la religión de la libertad de este autor y el socialismo liberal de Carlo Roselli. Sobre este tema escribí también artículos en periódicos y revistas de la emigración política italiana y tuve en fin, una viva discusión con Francisco Ayala que dio lugar a la publicación de un ensayo conjunto sobre el pensamiento político de las jóvenes generaciones italianas y españolas. Este ensayo apareció en 1944 en las «Jornadas del Colegio México» y de él me he vuelto a ocupar en un artículo reciente que suscitó una brillante intervención de Francisco Ayala publicada en El País de 25 de febrero de 1988.

3. Usted ha venido defendiendo siempre, en la línea de Weber y Kelsen, la tesis de la separación entre teoría jurídica y sociología del Derecho. En los últimos años, sin embargo, parece que asistimos a un cierto resurgir de las teorías del Derecho de impregnación sociológica: nos referimos, por ejemplo, a la propuesta bobbiana de una teoría del Derecho prevalentemente funcional y no ya estructural, a la teoría «institucional» del Derecho de Weinberger y MacCormick, a movimientos como los Critical Legal Studies, o a la insistencia de J. Raz en la necesidad de integrar en la teoría del Derecho algunos elementos capitales de la teoría política. ¿Qué opinión le merecen estas orientaciones? ¿Cree que pueden considerarse como indicativas de que la tesis de la separación está perdiendo fuerza?

Para contestar a esta pregunta debo recordar, ante todo, que la sociología del Derecho, a mi juicio, debe dividirse en dos partes que se ocupan de dos problemas diversos. Por un lado, del problema de la sociedad en el Derecho, es decir, de los comportamientos y opiniones de los individuos y de los grupos respecto al Derecho vigente, problema que hay que profundizar con investigaciones empíricas microsociológicas realizadas con instrumentos y métodos adecuados, y efectuadas teniendo en cuenta los desarrollos de la ciencia jurídica y el significado de las diversas direcciones que la caracterizan. Por otro lado, del problema general del Derecho en la sociedad, esto es, de la posición, de la 
función y del fin del Derecho en la sociedad visto en su conjunto; este problema exige investigaciones conectadas con la sociología general, la filosofía, la historia y la política.

De la separación entre teoría jurídica y sociología del Derecho a la que se refiere la pregunta, se ocupa la primera parte de la sociología del Derecho, y se trata de una separación que ha sido claramente especificada por un formalista como Kelsen, para el cual la teoría del Derecho se ocupa de las normas y la sociología del Derecho, de los hechos o, mejor, como él dice, de los fenómenos paralelos de la naturaleza que establecen, cumplen o violan las normas. Se trata además de una distinción hecha también por un sociólogo como Weber, quien hace notar que la teoría del Derecho se ocupa de las normas y del sentido lógicamente correcto que debe atribuirse a su expresión lingüística, y la sociología del Derecho, de los comportamientos, esto es, del actuar de los hombres referido a las normas y de las representaciones que estos mismos dan de las normas. Pero al citar el ejemplo de estos autores que han tenido el mérito de distinguir la teoría del Derecho de la sociología empírica del Derecho no pretendo sostener exclusivamente las concepciones de los formalistas en el ámbito de la teoría del Derecho. En este ámbito, comparto más bien la propuesta bobbiana de una teoría del Derecho prevalentemente funcional y no estructural y considero con interés la concepción institucional de Hauriou y de Romano así como la del Derecho como «regla de la vida social» de Duguit o la del Derecho como «hecho social» de Gurvitch. A estos y a otros autores formalistas les dirijo sólo el reproche de no haber distinguido la sociología del Derecho de la ciencia del Derecho entendida como jurisprudencia sociológica y de haber confundido la primera con la segunda. Una confusión como ésta era quizás admisible en los tiempos de Ehrlich e incluso en los de Pound, pero no es admisible hoy, en una época en la que estas investigaciones han alcanzado un desarrollo tal que no pueden ya ser consideradas como un simple capítulo de una disciplina jurídica cuyos cultores ignoran entre otras cosas las técnicas específicas de las referidas investigaciones.

No he hecho estudios específicos sobre los autores y direcciones de pensamiento a que se refiere esta pregunta y no estoy por tanto en condiciones de contestar en forma adecuada. De cualquier modo, puedo hacer notar que Raz, en el último capítulo de su libro The Concept of a Legal System acepta implícitamente la distinción kelseniana entre teoría formal y sociología empírica del Derecho, cuando, al afrontar el problema de la existencia de los sistemas jurídicos, propone tests que no pueden 
ser utilizados si no es sirviéndose de los instrumentos de la investigación empírica. En obras sucesivas, como The Morality of Freedom, demuestra un interés por problemas de filosofía política que pueden entrar también en el campo de la sociología teórica del Derecho.

En la última edición de mi libro Sociologia del diritto cito la obra de Weinberger y MacCormick con el simple fin de recordar que la obra de Hauriou despierta aún actualmente un vivo interés, y también con esta finalidad recuerdo los nombres de otros autores que, como Schelsky y Hagen, han retomado y desarrollado igualmente la teoría de la institución. Sobre el movimiento de los Critical Legal Studies me es difícil expresar un juicio. He leído algunos escritos de Unger que es uno de los autores más representativos de ese movimiento. Tengo la impresión de que el sociólogo del Derecho, empírico o teórico, puede extraer de ellos estímulos útiles para investigaciones de carácter histórico, antropológico o psicológico, además de jurídico o político, pero no indicaciones precisas sobre los problemas de metasociología del Derecho, relativas a los límites y a las tareas de la misma disciplina.

4. Usted se ha mantenido fiel hasta ahora a una concepción relativista de la ética a la que asocia, a la manera de Kelsen, con los valores de la democracia liberal. Sin embargo, el relativismo, como teoría metaética y también como instancia de fundamentación de los valores democrático-liberales aparece, por un lado, cada vez más puesto en cuestión y, por otro, la discusión ética parece centrada, al menos de quince años acá, en teorías éticas de carácter marcadamente cognoscitivista. ¿Cuál es actualmente su posición sobre estos temas? ¿Cree que puede seguir considerándose al relativismo como la mejor fundamentación posible de los valores democráticos?

En la respuesta a la segunda pregunta, he manifestado mi simpatía por el perspectivismo de Ortega y Gasset y por el problematicismo de Mondolfo, y en muchos escritos incluso recientes he elogiado el relativismo de Kelsen y de Radbruch, de manera que soy substancialmente contrario al cognoscitivismo, esto es, al procedimiento de derivación de los juicios de valor a partir de los juicios de hecho, que encuentra su aplicación más evidente en las doctrinas del Derecho natural que tratan de fundamentar objetivamente en los hechos los valores expresados en las mismas normas de comportamiento. Considero, sin embargo, que en la base de la distinción entre juicios de hecho y juicios de valor subyace un valor de importancia fundamental: el valor ex- 
presado por el principio de la tolerancia liberal que es propio de quien no pretende tener el monopolio de la verdad y muestra respeto, comprensión y disponibilidad hacia el pensamiento de los otros; el valor expresado por el principio del diálogo, según el cual, como dice Calogero, «es necesario que no sólo se toleren y se admitan las visiones de las cosas de los otros, sino que se demuestre por ellas una sincera curiosidad; es necesario que se tenga interés en comprender los modos mentales de los otros del mismo modo que se desea que los otros traten de comprender los nuestros»».

Con la referencia a este valor expresado por los principios de la tolerancia y del diálogo, considero que, aun no siendo cognoscitivista, puedo manifestar todo mi aprecio por las doctrinas iusnaturalistas y, en general, por las doctrinas cognoscitivistas en la medida en que sostengan y defiendan tales principios.

5. La rica sociología del Derecho italiana es, en muy amplia medida, un producto de su magisterio y de sus iniciativas a partir de finales de los 50. ¿Podría explicarnos cómo se produjo el inicial despegue de esa disciplina y qué etapas fundamentales cabe distinguir a partir de entonces? ¿Qué iniciativas e instituciones han jugado un papel más destacado? ¿Qué lecciones podemos extraer de la experiencia italiana para desarrollar en España la sociología del Derecho?

Para contestar a esta pregunta es necesario distinguir el problema del afianzamiento de la sociología del Derecho en la cultura jurídica italiana, del problema de la introducción de la enseñanza de esta materia en las universidades y en los planes de estudio de las diversas Facultades.

Por lo que se refiere al afianzamiento de la materia en nuestra cultura jurídica, el primer paso importante en tal sentido tuvo lugar con la investigación sobre la administración de justicia en Italia que se desarrolló bajo mi dirección en el ámbito de las actividades del «Centro nazionale di prevenzione e difesa sociale», entre el final de los años sesenta y los inicios de los años setenta, y que se realizó o sólo con la colaboración de jueces y de juristas, sino también con la de economistas, historiadores, sociólogos, investigadores empíricos y expertos en organización industrial. Esa investigación tuvo, indudablemente, el mérito de despertar interés por la materia en nuestra cultura jurídica; el interés se debía a la novedad que representaba, al prestigio y a la notoriedad de los estudiosos que participaban en ella y también al hecho de que los resultados alcanzados se recogieron en una 
colección de doce volúmenes ampliamente difundidos por una prestigiosa editorial. Pero la investigación fue también objeto de numerosas críticas. En 1968, cuando apenas habían salido los primeros volúmenes de esta colección, juristas de autoridad, reunidos en un congreso que tuvo lugar en Ancona, aun reconociendo el interés que las investigaciones empíricas podían presentar para su trabajo, advirtieron que tales investigaciones tendrían que haber sido dirigidas exclusivamente por juristas, reservando a los sociólogos y a otros expertos, únicamente funciones ejecutivas subalternas. En 1971, cuando los resultados de la investigación ya concluida se presentaron al congreso internacional de la disciplina que tuvo lugar en aquel año en Varese, los jueces de la corriente de «Magistratura democrática» criticaron el «cientificismo sociológico que realiza un continuo trabajo de ocultamiento de los momentos más espinosos de la realidad social» y hacían notar, por ejemplo, que el funcionamiento del aparato judicial sólo interesa a quienes pertenecen a la parte de la sociedad a cuyo servicio funciona dicho aparato. En 1972, durante el congreso que tuvo lugar en Catania sobre «L'uso alternativo del diritto», algunos juristas, aunque no refiriéndose directamente a nuestra investigación, criticaron indirectamente su orientación reformista, señalando que la función de la ciencia del Derecho debe ser una función revolucionaría dirigida a favorecer la emancipación económica y social de la clase trabajadora.

En 1974, a pesar de estas críticas y quizás precisamente a causa de las mismas, se efectuó el segundo paso decisivo para el afianzamiento de la sociología del Derecho en el ámbito de la cultura jurídica italiana. En base a un acuerdo entre el centro antes indicado y el «Istituto di filosofía del diritto» de la Universidad de Milán, entonces dirigido por mí, se decidió publicar la revista «Sociología del diritto», la primera y, hasta ahora, la única revista sobre esta materia en nuestro país. En el primer número no se presentó un programa, pero se abrió un debate sobre la naturaleza y los objetivos de la disciplina. En el debate participaron numerosos estudiosos italianos y extranjeros. Las opiniones manifestadas fueron muchas y diversas. No es posible examinarlas aquí con detalle. Se trata, por otro lado, de opiniones bien conocidas en España, por el amplio análisis que de ellas hizo en su momento Elías Díaz. Sólo diré que en ellas se manifiesta un evidente contraste entre quienes tendían a incorporar la disciplina en la ciencia jurídica, haciéndola entrar prácticamente en la jurisprudencia sociológica, y quienes trataban por el contrario de asegurar la autonomía de la disciplina, señalando la 
importancia de su vinculación con la sociología empírica, por un lado, y con la sociología teórica, por el otro.

El problema de la introducción de la enseñanza de la sociología del Derecho en las universidades y en los planes de estudio de las diversas Facultades es un problema en el que hay que profundizar teniendo en cuenta las situaciones personales, culturales, políticas y administrativas de cada país. En Italia, ciertamente, han ayudado las iniciativas que acabo de recordar, pero también han ayudado otros factores. Por ejemplo: el gran interés mostrado en estos últimos años por las materias sociológicas por parte de diversas Facultades y, especialmente, por las de ciencias políticas; además, la constitución de nuevas universidades y de nuevas facultades; $y$, en fin, la presencia de jóvenes activos y preparados, muchos de los cuales ocupan dignamente, ya desde hace algunos años, cátedras universitarias. Concluyo poniendo de relieve que, para la enseñanza de esta materia, las Facultades de Derecho son teóricamente las más adecuadas, pues en ellas se encuentran los jóvenes más interesados y preparados, pero son también las que están menos dispuestas a introducir cátedras de sociología del Derecho. Las Facultades de ciencias políticas, aun contando con estudiantes menos motivados, son, por el contrario, las más abiertas a acoger y favorecer las nuevas iniciativas. 